\title{
Ethnologies
}

\section{Entre psychopathologie et religion/spiritualité}

\section{Le sens "guérisseur »}

\section{Véronique Béguet}

Volume 33, numéro 1, 2011

URI : https://id.erudit.org/iderudit/1007803ar

DOI : https://doi.org/10.7202/1007803ar

Aller au sommaire du numéro

\section{Éditeur(s)}

Association Canadienne d'Ethnologie et de Folklore

ISSN

1481-5974 (imprimé)

1708-0401 (numérique)

Découvrir la revue

Citer cet article

Béguet, V. (2011). Entre psychopathologie et religion/spiritualité : le sens " guérisseur ». Ethnologies, 33(1), 219-238. https://doi.org/10.7202/1007803ar

\section{Résumé de l'article}

Cette note de recherche se situe à la confluence de deux types d'écrits en santé mentale. D'un côté, un nombre croissant de recherches questionnent la frontière entre la psychopathologie et l'expérience spirituelle ou religieuse. De l'autre, le sens donné à l'expérience est considéré essentiel au rétablissement des personnes ayant des troubles psychiatriques. Cette note présente les récits de deux personnes avec un diagnostic psychiatrique, des sens qu'elles ont donnés à leur expérience (puisant dans le mysticisme et la conversion spirituelle) et des manières dont elles les ont intégrées pour un rétablissement complet. L'expérience directe de dimensions invisibles du monde et les " savoirs » que les personnes en tirent sont fondatrices de leur repositionnement.
Ce document est protégé par la loi sur le droit d'auteur. L'utilisation des services d'Érudit (y compris la reproduction) est assujettie à sa politique d'utilisation que vous pouvez consulter en ligne.

https://apropos.erudit.org/fr/usagers/politique-dutilisation/ 


\title{
ENTRE PSYCHOPATHOLOGIE ET RELIGION/SPIRITUALITÉ
}

Le sens "guérisseur "

\author{
Véronique Béguet ${ }^{1}$ \\ Université du Québec à Trois-Rivières
}

Le sens donné à l'expérience est considéré comme un élément important du processus de rétablissement, terme que le champ de la santé mentale préfère à celui de " guérison ». La notion de rétablissement est issue de récits de «survivants ${ }^{2}$, d'études longitudinales et d'intervenants psychosociaux qui, tous, ont remis en cause la chronicité et le destin inéluctable de la «maladie mentale » (Rodriguez et al. 2006). Mal définie, elle apparaît comme une notion "fourre-tout ", à la fois ensemble de significations, valeur, paradigme et politique pour le changement (Bonney et al. Stickley 2008). Davidson et ses collaborateurs (2005) montrent la coexistence d'une perspective strictement clinique selon laquelle le rétablissement concerne la diminution ou la disparition des symptômes, d'une approche (je dirais politique) mettant l'accent sur le rétablissement de l'exclusion sociale, du stigma, des étiquettes, des restrictions, des droits civils, de la discrimination, de la culpabilité et de la honte (Bonney et Stickley 2008) et, enfin, d'un processus individuel. De ce dernier point de vue, le rétablissement est un cheminement profondément personnel de changement et de travail sur soi, sur ses attitudes, ses valeurs, ses sentiments, ses compétences et ses projets de vie qui aboutit à une vie

1. Cette note de recherche a grandement bénéficié des commentaires judicieux de Carl Lacharité, Deirdre Meintel, Géraldine Mossière et Travis, puis des évaluateurs externes. Je les remercie tous ici. Dans un souci éthique, le texte a été donné pour lecture aux participants concernés afin qu'ils puissent y réagir avant sa publication: Travis a corrigé certaines erreurs factuelles tandis que Nicolas a simplement donné son feu vert.

2. Le témoignage de Patricia Deegan (1988) constitue l'un des textes pionniers aux États-Unis. Au Québec, quelques personnes rétablies ont publié leur histoire (Dôle 2000 ; Langlois 2004,) ou l'utilisent lors de conférences (Vigneault). 
gratifiante et donne un sens nouveau à l'existence en dépit de la maladie et des symptômes éventuellement persistants (Corin 2002; Rodriguez et al. 2006 ; Lauzon et Lecomte 2002 ; Spaniol et al. 2002 ; Karlsonn 2009). La spiritualité participe très souvent de ce repositionnement (Spaniol et al. 2002) et le sens donné à l'expérience, comme vecteur d'une redéfinition identitaire, lui est crucial (Judge et al. 2008: 98 ; Spaniol et al. 2002 ; Corin 2002). Toutefois, ce sens est encore largement conditionné par les paradigmes médicaux et psychosociaux (Spaniol et al. 2002).

Dans leur étude pionnière portant sur 183 entendeurs de voix dont $48 \%$ sans problèmes psychiatriques, Romme et Escher (1993) ont souligné que le sens donné aux voix et la capacité de le partager socialement constituent un critère essentiel qui distingue les entendeurs de voix sains de ceux avec des troubles psychiatriques. En 1993, leur étude laisse place à de nombreuses interprétations (religieuses et mystiques, parapsychologiques, karmiques ou métaphysiques) tandis qu'en 2009, l'éventail interprétatif s'est rétréci et une perspective psychologique domine les 50 récits de rétablissement.

Cette note de recherche prolonge les conclusions des écrits sur le rétablissement et celles de Romme et Escher en mettant en exergue l'importance du sens donné à l'expérience dite hallucinatoire. Elle présente les récits de deux personnes hospitalisées en psychiatrie pour un épisode qu'elles considèrent dans une perspective mystique ou spirituelle. Elle s'inscrit dans les débats contemporains en santé mentale entourant la distinction du spirituel/religieux et du pathologique. Cependant, elle ne vise pas leur démarcation objective, mais se situe au contraire à leur frontière en explorant l'expérience et les sens qui lui sont donnés. Dans le sillage de l'anthropologie médicale, elle s'intéresse à la narration, à l'expérience subjective de la maladie et du sens qui lui est donné, à la réalité vécue.

\section{Le débat dans le champ de la santé mentale}

En 1994, sous l'impulsion de Lukoff, Lu et Turner (1992), une nouvelle catégorie est créée dans le DSM-IV (l'outil diagnostic nordaméricain et anglo-saxon en santé mentale) afin de distinguer l'expérience spirituelle et religieuse de la maladie mentale. Cette modification est majeure. Elle renoue avec la prise en compte du religieux en santé mentale qui, selon Dein (2005), a été progressivement 
occulté au XIX $\mathrm{X}^{\mathrm{e}}$ siècle pour devenir tabou durant la majeure partie du $\mathrm{XX}^{\mathrm{e}}$, à la fois en psychiatrie et en psychologie $\mathrm{e}^{3}$. Ce rejet est expliqué par le cadre de pensée positiviste, la difficulté à mesurer l'expérience religieuse et la déclaration de Freud concernant le caractère psychopathologique de celle-ci (Dein 2010 ; Heriot-Maitland 2008).

La forte prépondérance du spirituel et du religieux parmi les personnes présentant un trouble de santé mentale est attestée par de nombreuses études, le plus souvent quantitatives (voir par exemple la synthèse de Koening 2009). Huguelet et ses collaborateurs (2006) notent par exemple que la majorité des 100 participants à leur recherche (des personnes suivies en consultation externe pour schizophrénie) rapportent que la religion est une dimension importante de leur vie. En outre, $16 \%$ ont des hallucinations qui reflètent leurs croyances religieuses. Toutefois, seuls 36\% en parlent à leur psychiatre. Un grand nombre d'écrits psychologiques considèrent la religion et la spiritualité comme des stratégies adaptatives aux troubles mentaux, comme des ressources qu'il est possible de mobiliser à des fins de rétablissement (par exemple Huguelet et al. 2006 ; Koening 2009). En conséquence, des professionnels en appellent à l'implantation de soins spirituels (Culliford 2004 ; Coyte 2010 ; Durà-Vilà et Dein 2009).

Par ailleurs, les deux types d'expériences présentent de nombreuses similitudes (Eeles et al. 2003 ; Sanderson et al. 1999) et posent le problème de leur discrimination. Parmi les dénominations variées de l'expérience religieuse (mystique, extatique, sacrée, renaissance, paranormale, sortie hors corps, flux, transcendance et conversion) (Bartocci et Dein 2005), la transformation spirituelle et le mysticisme (et leurs différences d'avec la psychopathologie) retiennent particulièrement l'attention.

3. Comme le souligne un évaluateur, le spirituel n'a pas été totalement exclu de la psychologie durant cette période avec notamment l'apport de la psychologie humaniste dès les années 1950 (Rogers, Moreno, Lowen). Si les approches humanistes concernent également les personnes avec des troubles psychiatriques, elles ne se centrent cependant pas sur cette dimension. C'est pourquoi elles ne sont pas présentées ici. 


\section{La transformation spirituelle et le mysticisme}

L'intérêt pour la transformation spirituelle découle des travaux de Stanislas Grof et de son épouse Christina (1989) sur les états modifiés de conscience, qui sont à la source de la proposition de Lukoff et de ses collaborateurs (1998). Ces travaux participent de la psychologie transpersonnelle, intéressée au développement de la conscience, qui prend comme prémisse la nature profondément spirituelle de l'humain et la capacité de transcender les limites du corps-ego pour une appréhension plus vaste de la réalité ${ }^{4}$ (Collins 2008). Ce développement passe parfois par des crises, des changements brutaux et chaotiques que Grof et Grof (1989) ont nommés « spiritual emergency », jouant sur la double signification de «emergency» comme «émergence» et « urgence» (Grof 2002). Ces émergences spirituelles sont connues de diverses traditions : expériences mystiques, éveil de la kundalini ${ }^{5}$ dans les traditions orientales, initiations chamaniques, états de possession, expériences paranormales, ouverture psychique, spiritisme, expériences paroxystiques (Grof et Grof 1989; Grof 2002). Elles se caractérisent par de nombreux phénomènes tels que des visions, la sensation de présence énergétique, le fait d'entendre des voix, la perte de contact avec le monde matériel et la fusion avec l'univers, la connexion avec le monde animal et végétal, les mémoires passées, les sorties de corps, et la capacité soudaine de parler plusieurs langues (Collins 2008). En outre, les personnes peuvent ne plus fonctionner socialement durant ces crises spirituelles, ce qui aggrave les risques de diagnostic de troubles mentaux. La crise spirituelle toutefois amène une transformation profonde de la personne, voire sa guérison, et une conscience accrue par l'appréhension directe de la nature divine de la réalité. Grof (2002) précise que les états diagnostiqués comme psychose ne sont pas tous des émergences

4. Grof appelle « états de conscience holotropique » ces perceptions sensorielles profondément modifiées mais non altérées par des défaillances organiques, et induites par diverses pratiques incluant un travail sur la respiration, la méditation, la prière et autres pratiques spirituelles, les techniques sonores ou la danse, l'isolation sociale et sensorielle, le jeûne, les privations, les substances psychédéliques (Grof 2002).

5. Selon les yogis Indiens, la kundalini est une force cosmique qui, chez l'humain, se loge à la base de l'épine dorsale dans le corps subtil entourant et animant le corps physique. Lorsqu'elle est éveillée, cette énergie se déploie, comme un serpent, à travers tous les chakras (les centres d'énergie) et provoque leur ouverture (Grof 2002). 
spirituelles et peuvent effectivement relever d'une pathologie biologique.

Comme le rappelle Dein (2010), le mysticisme se caractérise par le sentiment fort d'une révélation, ineffable. L'expérience est transitoire, avec un retour rapide à la pensée sociale consensuelle (contrairement à la psychose) et au-delà de la volonté de la personne qui ne peut la susciter délibérément. Elle comporte une dissolution de la barrière entre soi et l'absolu et peut donner une vision de la réalité bien au-delà de ce que la critique rationaliste peut accepter (Dein 2010). Sa description se trouve dans les textes des mystiques religieux (notamment saint Jean de la Croix, sainte Thérèse D'Avila, saint François d'Assise), mais aussi dans les textes sur la folie ; toutefois, cette dernière est vécue négativement. Au-delà de ces différences, le contenu et la forme des expériences sont similaires, incluant des délires apparents, des changements radicaux de croyances, des hallucinations visuelles et auditives, des comportements étranges et le retrait social (voir Dein 2010 et les études sur lesquelles il s'appuie).

Durà-Vilà et ses collaborateurs $(2009$; 2010), démarquent « la nuit obscure de l'âme », expression provenant d'un poème du prêtre carmélite saint Jean de la Croix qui évoque la solitude et la désolation associée à crise spirituelle, ainsi que la dépression pathologique bien que toutes deux expriment les mêmes symptômes. Pour les personnes qui la traversent, cette nuit correspond à un processus de maturation spirituelle qui permet l'unification ultime et l'accès au véritable amour spirituel. Les auteurs concluent que le cadre interprétatif l'emporte sur les symptômes et assure une transformation de la vie pour le mieux (Durà-Vilà et Dein 2009). Ils confirment l'importance de la dimension subjective et interprétative de l'expérience, ce que reprennent les deux récits qui suivent.

\section{Les récits}

Les deux récits présentés ici sont issus d'une recherche postdoctorale ${ }^{6}$ en cours portant sur les expériences dites hallucinatoires de personnes ayant reçu un diagnostic psychiatrique et d'autres sans problèmes de

6. Cette recherche a été rendue possible par une bourse du Conseil de recherche en sciences humaines du Canada. Elle a reçu un certificat d'éthique de l'Université du Québec à Trois-Rivières. 
santé mentale, les sens qui leur sont donnés et leur intégration dans la vie des gens. Les participants ont été recrutés par bouche à oreille et devaient répondre aux critères d'avoir vécu des expériences dites hallucinations significatives et de leur avoir donné un sens (le type de significations étant laissé totalement ouvert). D'autres participants (dont le parcours n'est pas présenté ici) ont été rejoints par une invitation diffusée par le Réseau des Pairs aidants du Québec. En moyenne, deux entrevues semi structurées de deux heures chacune sont conduites avec chaque participant (au nombre de 21 au moment d'écrire ces lignes, parmi lesquels 6 avec un diagnostic psychiatrique). Ces expériences débordent largement le mysticisme ou la transformation spirituelle et couvrent une large gamme interprétative. Dans cette note toutefois, je m'en tiens à ces deux dimensions.

\section{Travis, 63 ans $^{7}$}

Travis est issu d'une de ces vieilles familles de la Nouvelle-Angleterre qui selon lui se considèrent comme le Peuple élu de Dieu. Protestante à l'origine, sa famille s'est convertie à la religion unitarienne à la génération de ses grands-parents, au moment où ce type de conversion était courant. Travis définit la religion unitarienne comme humaniste, rationnelle, s'enorgueillissant de célébrer le doute. La religion est un sujet important dans sa famille; son père, qui s'affirmait contre le dogme mais était respectueux des Églises, était d'ailleurs journaliste au Washington Post, spécialiste des religions. À treize ans, Travis est confronté à une religion chrétienne fondamentaliste du fait de ses voisins mormons. Il lit la Bible et de nombreux écrits religieux.

À seize ans, Travis connaît sa première expérience homosexuelle avec son compagnon de chambre dans une école privée prestigieuse. Aux États-Unis, à l'époque, l'homosexualité est une maladie mentale, un crime et un péché. Les autorités de l'école, mises au courant par son partenaire repentant, les séparent et leur imposent de voir un psychiatre. Pendant deux ans, Travis est torturé par les tentatives de son psychiatre de le guérir de cette maladie honteuse qui, selon lui, va ruiner sa vie, le priver d'emplois et d'amis. Travis considère que ces séances l'ont rendu fou, préparant le terrain à sa psychose à dix-huit ans et cette expérience traumatisante marquera à jamais ses rapports avec la psychiatrie.

7. Les prénoms sont fictifs, les âges sont ceux des personnes au moment de l'entrevue. 
À l'été 1963, à l'âge de dix-sept ans, Travis vit une extase. C'était

... ma première hallucination schizophrénique. Et c'était une vraie hallucination parce qu'en même temps, c'était aussi une vision béatifique. [...] Une extase. Une expérience religieuse comme ça, c'est ineffable, vous ne pouvez pas la décrire. Même avec des métaphores. Si je vous dis que mon âme est sortie de mon corps. Ce sont des mots. Parce que vous n'avez pas eu cette expérience. J'espère [rire]. Alors l'âme sort du corps et on atteint une extase. Vraiment. Une extase énorme. Et puis on voit. On voit le royaume de Dieu. Et c'est comme si un voile d'ange couvrait la face de Dieu. Parce qu'on ne voit pas Dieu. [...] Et on sent l'éternité. C'est ça. C'est l'éternité qu'on sent. C'est ça, la vérité suprême. [...] Une des connaissances que j'ai eues, c'est que ce monde dans lequel nous visons vous et moi, c'est ça l'illusion. C'est Dieu qui est réel. Et la vérité suprême, c'est que cette terre ici, notre monde à nous, est éphémère et passagère.

L'expérience ne dure que quelques secondes ou quelques minutes, mais elle est profondément transformatrice. Travis se promet de croire à cette hallucination jusqu'à la fin de sa vie : "J'ai eu le privilège de voir la vérité suprême, la vérité transcendante». De cette expérience, il garde une foi inébranlable. Mais que faire d'une telle expérience? Comment servir Dieu? Et vers qui se tourner pour le lui expliquer et le guider?

À dix-huit ans, il découvre que Paul Tillich, l'un des théologiens les plus influents de l'époque, vient donner un sermon à Boston. En Tillich, il voit un semblable, le seul à pouvoir comprendre et expliquer son illumination. Il rédige en hâte un essai de vingt-sept pages décrivant sa vision béatifique qu'il utilise comme preuve phénoménologique de l'existence de Dieu. Il fait lire son essai à Tillich qui lui propose, dans un sourire complice, de revenir le voir lorsque celui-ci sera achevé. Travis rentre chez lui et se met frénétiquement à finaliser ce texte qu'il pensait pourtant achevé. C'est là qu'il dérape. Alors que le premier texte est sain et articulé, le second est " un délire total », de plus en plus confus, reflétant l'état d'esprit dans lequel rentre le jeune homme. Deux jours après, un de ses amis, très inquiet, alerte les autorités de Harvard où il étudie alors et Travis est emmené de force à l'hôpital.

Commence alors cette période sombre de sa vie qui va durer quinze mois et qu'il associe à sa "nuit obscure de l'âme ${ }^{8}$ ». Le premier mois,

8. Rappelons que, selon Durà-Vilà et ses collaborateurs (2009, 2010), cette nuit obscure de l'âme renvoie plutôt à des symptômes dépressifs que psychotiques. 
Travis est toujours très confus, dans un délire psychotique dans lequel se côtoient des idées sexuelles bizarres, une paranoïa totale et des composantes mystiques. Il est en outre assommé par une médication puissante. Après un mois, Travis redevient lucide et se dit qu'il est à l'hôpital à cause d'un psychiatre et de la fragilisation de son être par les atteintes répétées à son identité sexuelle profonde. Sa première résolution est de survivre, de sortir de cet hôpital, de vivre sa vie selon ses propres critères, une vie libre. Cette décision témoigne de l'audace et de l'assurance de Travis, face à des psychiatres qui tenteront de lui asséner des vérités contraires. Ils lui répètent qu'il est totalement fou, l'un des pires qu'ils aient vu, qu'il n'aura jamais une vie normale, qu'il devrait renoncer immédiatement à son projet d'étudier à Harvard. Mais Travis est convaincu du contraire et ne partagera jamais ses motifs et ses expériences avec les psychiatres. "J'avais raison. J'étais assez intelligent pour savoir la cause de ma psychose. Et la solution». Le temps lui donnera raison.

Travis tient son assurance de ses connaissances théologiques et insiste sur le fait que les psychiatres, ignorant tout de la théologie, n'ont pas les compétences pour poser un jugement sur son illumination. Cette dernière est en outre une source de confiance, car

s'Il [Dieu] s'était révélé à moi, c'était un signe qu'il allait me soutenir et que je pourrais compter sur lui. Alors je crois, j'ai une certaine foi qui m'a permis de faire des choses merveilleuses dans la vie. Parce que cette foi en Dieu est aussi une forme de confiance en soi-même.

Ainsi, sa première force lui vient d'avoir tenue pour vraie cette expérience que les psychiatres qualifiaient de délire. Il est également soutenu par sa mère qui, malgré sa connaissance du pronostic défavorable lié à la schizophrénie du fait de sa formation d'infirmière, l'encourage à vivre sa vie et son homosexualité. Elle lui a raconté, lorsqu'il avait treize ans, que son oncle paternel, un homme qu'il admire profondément, a connu un épisode schizophrénique dans sa jeunesse. Il a été hospitalisé, mais s'en est sorti totalement et a eu par la suite une vie riche et influente. Cette histoire et son issue heureuse le confortent

Mais pour le jeune homme de dix-huit ans, son questionnement concernant Dieu est bien au cœur de sa crise et l'hospitalisation constitue la période la plus sombre de sa vie. 
dans sa certitude et sa résolution de s'en sortir lui aussi. À l'hôpital, il noue également une amitié très forte avec une autre patiente qui l'aide à vivre ces mois d'enfermement. Enfin, le dernier coup de pouce viendra de l'assurance médicale de ses parents qui, après treize mois, ne veut plus couvrir les frais d'hospitalisation. Travis est transféré dans un autre hôpital où on lui offre la possibilité de cesser sa médication. Il accepte et deux mois plus tard, il est enfin libre.

Sa première décision est d'oublier le mysticisme et «ces folies qui l'ont conduit à la psychiatrie » et de vivre sa vie. Il s'inscrit à l'Université Harvard et vit à Harvard square, le quartier hippy de Boston à l'époque. Là, l'homosexualité et l'expérience psychiatrique ne sont pas des handicaps, même plutôt un attribut seyant d'une jeunesse qui conteste la guerre du Vietnam et vit l'époque du Peace and Love. Il y noue des amitiés et des relations amoureuses bisexuelles fortes et il est entouré de gens qu'il aime et qui l'aiment. Citant la leçon principale de la Bible, Travis insiste sur le fait que « c'est l'amour qui guérit », pas les pilules. Pourtant, en dépit de cette vie heureuse, Travis, une fois son diplôme en poche, veut quitter les États-Unis. Officiellement, il part pour protester contre la guerre du Vietnam, mais au fond de lui, il sait qu'il veut mettre de la distance avec son passé psychiatrique.

Les huit années suivantes vont se dérouler dans différents pays d'Europe. Travis dira «qu'il est plus facile d'être étranger là où on est vraiment étranger ». Là, sa folie et sa marginalité passeront plus facilement inaperçues. Ainsi, c'est l'ouverture sur le monde et sur les autres, « à la fois paradoxale et salutaire pour un schizophrène », qui va parachever cette guérison. Il connaîtra une rechute, brève. Il migre finalement au Québec où il obtient un doctorat et travaille depuis. Au moment de l'entrevue, il est en couple avec un homme depuis treize ans et vit dans une petite ville.

Il oscille d'ailleurs entre rationalisme et mysticisme. Du premier, il apprécie les idées et l'univers intellectuel. Le second lui a donné la foi et la prière qu'il n'a jamais interrompue. Il assiste à la messe à certaines périodes, encore qu'il soit très critique de l'Église comme institution. Il a découvert avec le temps que certains choix qu'il a faits dans sa vie étaient liés à Tillich (par exemple d'aller vivre en Allemagne).

Travis souligne que son expérience est qualifiée « d'hallucination schizophrénique » par la psychiatrie moderne et de «vision béatifique » par le mysticisme chrétien. Il insiste sur le fait que l'expérience reste la 
même, les cadres interprétatifs changent selon les époques. Et s'il accepte le vocabulaire contemporain, il ne porte pas de jugement de valeur sur lui. Il note que la schizophrénie peut aussi être une expérience fondatrice, riche de sens et de créativité. Il marque une différence néanmoins entre l'épisode qui l'a conduit à l'hôpital, de «folie » perceptible par l'entourage, et l'illumination après laquelle il reste normal pour les autres. Sur le plan personnel, Travis n'est pas fier de la première et souhaiterait l'oublier, tandis que la seconde est pure, belle, extatique. Mais toutes deux sont du même ordre et s'inscrivent dans le même champ interprétatif. Dans le cadre de la psychiatrie, il a réussi sa schizophrénie comme en témoigne son parcours. Dans le cadre du mysticisme, il a vécu deux phases (l'illumination et la noirceur) d'une expérience globale qu'il a dépassée pour parvenir à cette vision unifiée qui intègre la foi et le rationalisme.

Travis n'en a pas totalement fini avec son expérience : certaines questions mystiques le taraudent parfois, notamment l'annonce de l'Apocalypse et la venue d'un Messie. Son point de vue étant "hérétique » par rapport au christianisme, il peut difficilement se rapprocher des théologiens contemporains. Conscient de l'anti-religiosité d'un Québec qui se déprend encore de la Grande noirceur, il se montre discret sur sa culture religieuse et sur sa foi. Alors il garde quelques certitudes pour lui et poursuit sa vie.

\section{Nicolas, 43 ans}

Nicolas, né en 1967, grandit entre un père «anti-soutane » et une mère catholique pratiquante. Il accompagne sa mère à la messe et apprécie l'atmosphère générale de l'église bien que le dogme et les rites lui importent peu. Il y développe un lien avec le sacré et peut "partir » dans ses pensées et ses prières, dans un mouvement intérieur qui le régénère et le purifie (Nicolas n'utilise pas ce terme, mais indique qu'il sortait de la messe comme d'une ablution). Il cesse d'accompagner sa mère à l'adolescence, préférant l'appartenance au groupe de pairs.

Nicolas expérimente très jeune des sorties hors corps. À quatre ans, alors qu'il est éveillé sur son lit, il voit soudain une sorcière qui l'emporte sur son balai et lui fait faire des tours de la pièce. Des expériences de déplacement astral (avec une dimension éthérique de l'humain, alors que le corps ne bouge pas) dans la maison durent le temps de son enfance et s'interrompent à l'adolescence pour reprendre 
alors qu'il est jeune adulte dans une période de stress. Le voyage astral lui donne des sensations très physiques : il sent la vibration dans ses cellules lorsqu'il quitte son corps et se sent passivement transporté vers la porte de sa chambre, la cuisine, le plafond par exemple. Avec le temps, il apprend qu'il peut contrôler ses déplacements par la pensée, sans jamais provoquer le phénomène qui, lui, est totalement involontaire. Lors des voyages astraux, le corps est dans une forme de paralysie ${ }^{9}$. Nicolas ne trouve personne à qui parler de ses expériences et tente de trouver des réponses dans les livres portant sur le surnaturel (encore qu'aucun à l'époque ne mentionne les sorties hors corps) et la vie après la mort. Il lit également la Bible. Cependant, aucune de ses lectures ne le prépare à vivre ce qui va suivre.

À vingt-quatre ans, un avenir prometteur s'ouvre à lui : une magnifique fiancée, un emploi important, une voiture rutilante nouvellement acquise. En quelques mois, cet avenir s'effondre et il est précipité dans l'abîme. Ses fiançailles sont rompues, il doit gérer la situation financière d'une entreprise qui va finalement sombrer dans la faillite, dans laquelle son père avait investi de fortes sommes, et il est enfin extrêmement préoccupé par l'attitude de son père face à sa mère, et ses infidélités (il a des rêves du Jugement dernier de son père par exemple). Durant cette période, il alterne entre la douleur morale liée à ces difficultés et des états de grande félicité.

Avec le recul, Nicolas s'aperçoit que les périodes de stress et de surmenage stimulent les expériences non ordinaires qu'il connaît. Dans ce cas, la tension est extrême. Un soir, au volant de sa voiture, il se met à prier intensément. Peu après, il fait un rêve dans lequel il se sent d'abord piteux à cause d'orgies sexuelles et change totalement d'état d'esprit en ressentant une présence qui touche son front avec le sien. Nicolas connaît deux sensations physiques. La première est celle de l'extase liée à la rencontre avec un être spirituel, une expérience qu'il a vécue à plusieurs reprises et qui est "comme deux énergies qui fusionnent ». La seconde, pas désagréable, ressemble à une opération au front. Après cette nuit-là, une migraine persistante disparaît et toute la douleur morale, la «déprime », la culpabilité et la sensation « d'être plus ou moins à côté de sa vie » s'effacent. L'expérience est puissante et transformatrice. Il adopte dès le lendemain un mode de vie très sain,

9. Il apprend bien plus tard qu'un oncle paternel avait parfois de telles sensations qu'il appelait « le lourd». 
voire ascétique, abandonne l'alcool, les gras, le sexe et se défait rapidement de ses possessions matérielles. "C'est mon corps qui me le demandait. Énergétiquement, ça me le demandait». À son réveil ce matin là, " je n'étais pas en dépression ; j'étais comme un cœur ouvert ».

Commence alors ce que Nicolas appelle sa neuvaine mystique, un état de félicité intense et de joie de vivre. Il se découvre de nouvelles facultés sensitives. Il ressent l'isolement, la solitude et le manque d'amour d'une auto-stoppeuse. Il lui communique de manière télépathique que Dieu l'aime et répète son message jusqu'à ce que, soudain, la jeune fille se transforme: ses masques tombent, ses joues ruissellent. (Dans un état normal, cette stratégie ne fonctionne pas, il l'a testée plus tard). Au même moment, un autobus scolaire passe avec des enfants qui leur font de larges sourires. À l'Oratoire Saint-Joseph, dans la chapelle SaintAndré, lui revient le projet de travailler pour aider les sans-abri, un projet qu'il a toujours repoussé du fait de la faible rémunération. Cette fois, il se sent au contraire libre et serein, sans peur de l'insécurité financière, avec une grande soif de vivre sa vie, quitte à le faire dans la différence. Il va à la rencontre des itinérants, crée des liens, entend leur histoire. Il lit dans un livre qu'il peut demander des leçons de sagesse avant de s'endormir et en reçoit une chaque nuit avec réveil à $4 \mathrm{H} 44$ pour qu'il puisse s'en souvenir et l'intégrer. Une de ces leçons l'incite à suivre son cœur pour trouver le but de son existence et de croire à la loi de l'attraction (attirance des situations favorables lorsqu'une intention est clairement manifestée). Dans un rêve, il vit l'éclatement de son ego pour laisser place au «Je» dans toute sa splendeur. À aucun moment cependant, il ne pense être un élu de Dieu ou l'incarnation d'une figure catholique légendaire. En fait, ses sorties de corps passées l'ont déjà ouvert à des possibilités non ordinaires et l'ont rendu humble face à elles.

Cette joie est assombrie par des périodes d'angoisse, lorsqu'il est seul chez lui et qu'il ressent de la culpabilité face à ses parents qui vont mal. Il lui arrive d'être terrorisé par un orage, une fenêtre qui claque, et de se blottir dans son lit. À quatre heures du matin, le téléphone sonne, il décroche et il entend des voix métalliques, presque démoniaques. Son entourage s'inquiète pour lui et tente de l'aider. Une tante religieuse avec laquelle il a beaucoup d'affinités l'appelle, l'écoute et le réfère à un prêtre. Ce dernier lui offre une séance de reiki (une forme de canalisation et de transmission d'énergie sans contact direct) au cours de laquelle il sent une énergie très forte autour du sexe. Après la séance, 
un élan le pousse à embrasser le prêtre pour un échange de souffle de vie, un mouvement qu'il interrompt rapidement et s'excuse, confus d'une éventuelle homosexualité. Sur ce plan, il sera rassuré peu de temps, en rompant son vœu de chasteté avec une nouvelle voisine. Avec le recul, Nicolas considère avoir échangé un baiser tantrique avec le prêtre et déclenché son ouverture sexuelle, sur le plan énergétique, avec sa voisine. Selon lui, cette ouverture, combinée avec celle du cœur qui a précédé, va permettre la montée de la kundalini.

Peu après ces événements, Nicolas a une vision, «le film de la création ». Il voit d'abord une énorme boule de lumière palpitant d'amour dans le néant. Par intuition mystique, il comprend qu'il s'agit du premier jour de la création et, dans son cœur, il ressent l'amour de Dieu. Sa voix intérieure lui enjoint de se calmer. Alors le Dieu de sa vision expulse le germe de l'Univers et le film zoome sur la terre. Des révélations sont transmises à Nicolas par le haut de sa tête. Au début, elles sont simples et il les note. Mais rapidement, leur rythme s'accélère et elles deviennent de plus en plus complexes, avec des équations mathématiques. Il voit ensuite l'avènement de l'Homme et un flux ininterrompu circule de Dieu vers celui-ci vers la matrice de la terre et tous les êtres, animaux, végétaux, cours d'eau qui la peuplent. Or Nicolas ne voit pas seulement cet homme nouvellement créé : il l'est. Et les mots lui manquent pour poursuivre sa description. Il est subjugué par une décharge d'amour, en fusion avec Dieu comme il l'a été lors de sa première extase. Il sent ce "flux trop puissant pour ses neurones » et, incapable de l'arrêter, il panique. Il appelle son père et lui demande de lui parler, de n'importe quoi, de sport.

Son père s'exécute et, terriblement inquiet, lui donne rendez-vous à Montréal. Nicolas prend le volant en ayant perdu tous ses repères et avec des facultés motrices altérées. Totalement désorienté, il finit par faire appeler son père pour lui demander de venir le chercher. Ce dernier le ramène à la maison familiale. Il y passe quinze jours, inerte et sans force. Finalement, il entend qu'un centre pour sans-abri est menacé de fermer et décide de lui apporter son aide.

Là, il fait la connaissance de Franciscains qui œuvrent auprès des itinérants et l'hébergeront quelques jours. En serrant dans ses bras un itinérant amnésique, son cœur se rouvre soudainement. La croix d'un prêtre à proximité et l'évocation de saint François provoquent une décharge électrique de tout le corps qui se termine en une "extase et 
une vision », la dernière, d'une longue chaîne d'hommes et de femmes, du sommet à la base, sans oublier le plus petit. Il est empli d'un nouvel élan de joie et s'effondre en larmes auprès de l'itinérant.

Les états de félicité alternent avec l'anxiété et un soir, il pense se jeter par la fenêtre. Il se rend à l'hôpital psychiatrique. Là, il vit une "nuit de l'âme », une sorte de «trépas psychique » avec un cour fermé, déserté par les sentiments élevés. Il reçoit un diagnostic de troubles bipolaires dans lequel il ne se reconnaît pas et refuse la prescription médicamenteuse, malgré les menaces de son psychiatre qui lui prédit des psychoses et des hospitalisations à répétition. Il accepte un anxiolytique qu'il prend un temps, à très petite dose au besoin. Après sa sortie, il lui faudra environ dix-huit mois pour retrouver son énergie et reprendre pied dans sa vie.

L'expérience de Nicolas a été tellement forte qu'il s'y est complètement abandonné. Mais elle l'a conduit à l'hôpital psychiatrique, " une claque » énorme où il a risqué de se désintégrer et a vécu le désespoir. À partir de là, il ne veut plus vivre cette ouverture du cœur et fait des choix qui l'ancrent dans la vie matérielle avec par exemple un emploi très rémunérateur dans le domaine de la communication. Néanmoins, il ne succombe jamais au matérialisme et privilégie des expériences professionnelles qui le nourrissent (quittant par exemple cet emploi lorsqu'il estime n'être plus motivé que par le salaire).

Nicolas découvre les écrits de Stanislav Grof sur l'émergence spirituelle quinze ans après son hospitalisation et se reconnaît immédiatement. Il a connu les épisodes de pression crânienne désagréables, les perceptions de sons internes et d'une lumière intérieure, les mouvements thermiques au sein du corps, l'anxiété qui l'habite lorsqu'il se retrouve seul chez lui, le sentiment de dépersonnalisation, les états de transes et de conscience paranormale, l'accélération de la pensée. À cette liste s'ajoutent la réduction des besoins physiques en termes de sommeil et de nourriture et l'accès à de grandes révélations. L'expérience a été puissante et transformatrice avec une augmentation de son sens moral et humaniste. Il découvre que la transmission de connaissance sous forme d'idées élevées, de théorèmes mathématiques et de symboles archétypaux a été décrite par Krishna, la montée de la kundalini par le Livre des morts tibétain. Nicolas réalise également que tous les saints ont connu ces fulgurantes ouvertures du cœur, le fantasme de sauver les âmes de l'enfer, l'impulsion de l'ascétisme et les visions 
transformatrices. Il comprend le caractère initiatique de l'expérience, la mort pour mieux renaître et intégrer de nouvelles dimensions à sa vie. Ce constat le sort de la stigmatisation et lui donne une autre perspective que celle d'avoir été victime d'une hospitalisation psychiatrique.

Soudain le sens de son expérience, qui jusque-là était un faux pas honteux connu de quelques intimes uniquement, lui apparaît et sa vie prend une nouvelle dimension. Il intègre la spiritualité à l'engagement social et travaille d'abord dans un organisme communautaire pour les itinérants, puis dans un autre en santé mentale. Il ressent à nouveau cette ouverture du cœur et l'accepte, d'autant qu'il s'y sent en sécurité et non plus menacé de "décoller». Cette ouverture nourrit profondément sa nouvelle relation amoureuse, son ancrage dans la famille recomposée à laquelle il appartient maintenant et elle lui permet une véritable empathie dans les milieux où il œuvre. Toute sa vie s'en trouve considérablement améliorée avec un ancrage dans la vie physique, corporelle, quotidienne et sociale plus fort. Sexuelle aussi puisque l'ouverture qu'il a connue a été nourrie par la suite par ses relations suivantes. Nicolas est critique des personnes qui ne vivent que dans la spiritualité. Pour lui, l'ancrage dans la vie est important.

L'idée là-dedans, c'est que la vie est là. J'en ai des frissons en te le disant. C'est tellement beau. C'est tellement une expérience enrichissante [celle de la vie]. Mais il faut que tu la vives, il faut que tu y goûtes. Mais quand tu peux y goûter avec la notion qu'il y a quelque chose ailleurs, puis que tu vibres, là tu es connecté. Et tu peux vraiment rendre ta vie utile pour d'autres et pour toi. [...] À ce moment-là, tu as une spiritualité qui est vibrante.

Nicolas souligne le côté intime, ineffable, de l'expérience. L'avoir expérimentée lui donne non seulement la foi, mais pratiquement un savoir. Il sait qu' « il y a autre chose », sans pouvoir le définir précisément. C'est comme d'être à l'orée d'une forêt. On sait qu'elle existe, mais on ne l'a pas parcourue. Dans tous les cas cependant, il souligne la grande distance qui sépare de tels savoirs avec le paradis dépeint par la religion chrétienne. 


\section{Conclusion}

Dans ces récits, l'expérience est ambivalente, source d'une hospitalisation traumatisante et fondatrice à la fois. Elle est considérée comme une maladie mentale par la psychiatrie et les psychiatres qui vont tenter de convaincre leurs patients de leur diagnostic. Ils s'appuient pour cela sur des constats "objectifs" : il existe effectivement des comportements problématiques, des "symptômes », une zone de « dérapage » qui conduit potentiellement à l'hospitalisation, ce dont Nicolas et Travis sont conscients. C'est d'ailleurs pour cette raison que l'un et l'autre décident de se distancier de leur expérience pour éviter les risques de rechute. Ils ne sont donc pas nécessairement dans un état de déni, qualificatif généralement accolé aux personnes qui ne se plient pas aux prescriptions médicales. Pourtant, tous deux sont convaincus que le diagnostic psychiatrique est erroné et ils refusent la médication qui l'accompagne. L'histoire va leur donner raison et, contrairement aux prédictions psychiatriques, ils ne seront plus hospitalisés. Ces deux récits n'invalident pas les descriptions objectives de la maladie mentale. Ils ne signifient pas, évidemment, que tous les diagnostics de délires mystiques par exemple sont erronés. Ils confirment pourtant la fragilité et la complexité du diagnostic psychiatrique et témoignent, comme le demandent Lukoff et ses collaborateurs (1998), en faveur d'une plus grande prudence et circonspection.

Ce n'est pas dans les «faits ", mais dans le sens qui leur est donné que se joue le débat. En effet, Travis et Nicolas ont accepté leur " dérapage ", le comportement visiblement "non ordinaire », mais ils lisent ou relisent leur expérience dans une autre perspective interprétative, respectivement mystique et spirituelle. Une perspective qui est essentielle à leur rétablissement : elle constitue une des clés de la résilience de Travis à l'hôpital et provoque un changement qualitatif dans la vie de Nicolas quinze ans plus tard. L'expérience n'a pas changé ; le sens qui est lui donné varie.

Plus important encore, cette perspective interprétative alternative est ancrée dans leur expérience. Intégrée sous des formes positives, cette dernière est source d'une très grande force et fonde la foi et la confiance inébranlables de Travis ainsi que la spiritualité incarnée de Nicolas. Le sens «guérisseur» n'est ni abstrait ni mental, ni le fruit de croyances ou de représentations sociales simplement acquis ; il est inscrit dans l'expérience individuelle. Il possède la profondeur, la force et la 
puissance interprétative que confère l'expérience directe, ineffable et résolument individuelle. C'est cette capacité à se relier à cette expérience, à en extraire et en intégrer le sens qui est guérisseuse. Ces résultats sont cohérents avec les conclusions de Romme et Escher (1993) sur l'importance du sens comme facteur départageant les entendeurs de voix avec ou sans problèmes psychiatriques et, de manière générale, avec l'appel général à la prise en compte du sens dans le processus de rétablissement. Or c'est précisément cette tentative qui est systématiquement cassée dans le milieu psychiatrique où elle est qualifiée de déni, de non-acceptation de la maladie mentale.

Enfin, pour Travis et Nicolas, cette expérience donne accès à un savoir différent de celui du rationalisme et du matérialisme, un savoir sur le monde, sa création et sa dimension divine. Ce savoir les conforte dans leur posture même si elle s'inscrit en porte-à-faux avec la psychiatrie et le rationalisme dominants et il met en scène des dimensions longtemps occultées de l'humain et du monde. 


\section{Références}

Bartocci, Goffredo et Simon Dein, 2005, «Detachment: Gateway to the World of Spirituality?». Transcultural Psychiatry 42 (4) : 545569.

Bonney, S. et Theodore Stikeley, 2008, « Recovery and Mental Health: A Review of the British Literature ». Journal of Mental Health and Psychiatric Nursing 15: 140-152.

Collins, Mick, 2008, «Politics and the Numinous: Evolution, Spiritual Emergency, and the Re-emergence of Transpersonal Consciousness ». Psychotherapy and Politics International 6 (3) : 198-211.

Corin, Ellen, 2002, «Se rétablir après une crise psychotique. Ouvrir une voie ? Retrouver sa voie?». Santé mentale au Québec 27 (1): 65-82.

Coyte, Mary-Ellen, 2010, "The Quest». Mental Health, Religion 8 Culture 13 (6) : 553-565.

Culliford, Larry, 2002, «Spiritual Care and Psychiatric Treatment: An Introduction ». Advances in Psychiatric Treatments $8: 249-258$.

Davidson, Larry, Maria J. O'Connel, Janis Tonora, Martha Lawless et Arthur C. Evans, 2005, «Recovery in Serious Mental Illness: A New Wine or Just a New Bottle? ». Professional Psychology: Research and Practice 36 (5) : 480-487.

Deegan, Patricia E., 1988, «Recovery: The Lived Experience of Rehabilitation ». Psychosocial Rehabilitation Journal 11: 11-19.

Dein, Simon, 2010, "Judeo-Christian Religious Experience and Psychopathology: The Legacy of William James ». Transcultural Psychiatry 47 (4) : 523-547.

__, 2005, «Spirituality, Psychiatry and Participation: A Cultural Analysis ». Transcultural Psychiatry 42 : 526-544.

Dôle, Robert, 2000, Comment réussir sa schizophrénie. Montréal, VLB éditeur.

Durà-Vilà, Gloria, Simon Dein, Roland Littlewood et Gerard Leavey, 2010, "The Dark Night of the Soul: Causes and Resolution of Emotional Distress Among Contemplative Nuns ». Transcultural Psychiatry 47: 548-570.

—_ et Simon Dein, 2009, «The Dark Night of the Soul : Spiritual Distress and its Psychiatric Implications ». Mental Health, Religion E3 Culture 12 (6) : 543-559.

Eeles, Jennie, Trevor Lowe et Nigel Wellman, 2003, «Spirituality or Psychosis? An Exploration of the Criteria that Nurses Use to 
Evaluate Spiritual-type Experiences Reported by the Patients ». International journal of nursing studies 40 : 197-206.

Grof, Stanislav, 2002, Pour une psychologie du futur. Transformation psychique et paix intérieure. Paris, Éditions Dervy.

_- et Christina Grof, 1989, Spiritual Emergency: When Personal

Transformation Becomes a Crisis. Los Angeles, J.P. Tarcher.

Hériot-Maitland, Charles P., 2008, « Mysticism and Madness: Different

Aspects of the Same Human Experience? ». Mental Health, Religion

Eु Culture 11 (3) : 301-325.

Huguelet Philippe, Sylvia Mohr, Laurence Borras, Christiane Gillieron et Pierre-Yves Brandt, 2006, "Spirituality and Religious Practices Among Outpatient With Schizophrenia and their Clinicians ». Psychiatric Services 57 (3) : 366-372.

Judge, Abigail M., Sue E. Estroff, Diana O. Perkins et David L. Penn, 2008, "Recognizing and Responding to Early Psychosis: A Qualitative Analysis of Individual Narratives ». Psychiatric services 59 (1) : 96-99.

Karlsson, Lis Bodil, 2009, "Schizophrenic or Occult Harassed?». Qualitative Social Work 8 (1) : 83-100.

Koening, Harold G., 2009, « Research on Religion, Spirituality and Mental Health: A Review ». The Canadian Journal of Psychiatry 54 (5) : 283-291.

Langlois, Richard, 2004, Le fragile équilibre. Montréal, Le Dauphin blanc. Lauzon, Gilles et Yves Lecomte, 2002, «Rétablissement et travail ».

Santé mentale au Québec 27 (1) : 7-34.

Lukoff, David, Francis Lu et Robert Turner, 1998, «From Spiritual Emergency to Spiritual Problem: The Transpersonal Roots of the New DSM-IV Category ». Journal of Humanistic Psychology 38 : 2150 .

- - 1992, "Towards a More Culturally Sensitive DSM-IV: Psychoreligious and Psychospiritual Problem ». Journal of Nervous and Mental Disease 180 (11): 673-682.

Rodriguez, Lourdes, Linda Bourgeois, Yves Landry, Lorraine Guay et Jean-Luc Pinard, 2006, Repenser la qualité des services en santé mentale dans la communauté. Changer de perspective. Montréal, PUQ.

Romme, Marius et Sandra Escher, 1993, Accepting Voices. Londres, Mind Publications.

__, 2009, Living With Voices: 50 Stories of Recovery. Londres, Mind Publications. 
Sanderson, Susan, Brian R. Vandenberg et Paul Paese, 1999, «Authentic Religious Experience or Insanity?». Journal of Clinical Psychology 55 (5): 607-616.

Spaniol, Leroy, Nancy J. Wewiorski, Cheryl Gagne et William A. Anthony, 2002, "The Process of Recovery From Schizophrenia». International Review of Psychiatry $14:$ 327-336. 